TENDENCIAS

Revista de la Facultad de Ciencias

Económicas y Administrativas.

Universidad de Nariño

ISSN-E 2539-0554

Vol. XXII No. 2 - 2do Semestre 2021

Julio-Diciembre - Páginas 1-25

\title{
PERCEPCIÓN DEL CONSUMIDOR SOBRE EL IMPACTO DEL MERCHANDISING OLFATIVO COMO ESTRATEGIA DEL MARKETING SENSORIAL
}

CONSUMER PERCEPTION OF THE IMPACT OF OLFACTORY MERCHANDISING AS A SENSORY MARKETING STRATEGY

\section{PERCEPÇÃO DO CONSUMIDOR SOBRE O IMPACTO DA COMERCIALIZAÇÃO OLFATIVA COMO ESTRATÉGIA DE MARKETING SENSORIAL}

\author{
Claudia Patricia Grisales Castro; Edwin Arbey Hernández García; \\ Evelyn Xiomara Montenegro Cardona
}

Magíster en Marketing, Universidad Libre, Colombia. Docente investigativo, Facultad de Comunicación, Corporación Universitaria para el Desarrollo Empresarial y Social (CUDES). ORCID: 0000-0002-8881-3836. E-mail: claudia.grisales@ cudes.edu.co, Colombia.

Magíster en Economía Aplicada, Universidad del Valle, Colombia. Docente e investigador de la Corporación Universitaria para el Desarrollo Empresarial y Social (CUDES). Integrante del Grupo de Investigación de Modelación Matemática y Estadística para las Ciencias Sociales (GIMMECS). ORCID: 0000-0002-5919-7659. E-mail: edwinarbeyh@gmail.com, Colombia.

Profesional en Marketing y Negocios Internacionales, Corporación Universitaria para el Desarrollo Empresarial y Social (CUDES). 0000-0002-7678-5225. E-mail: evelynmontenegrocar@gmail.com, Colombia.

Recibido: 21 de agosto de 2020

Aprobado: 18 de enero de 2021

DOI: https://doi.org/10.22267/rtend.212202.166 


\title{
Resumen
}

La percepción de olores siempre ha estado relacionada con el comportamiento del ser humano, sin embargo, como estrategia empresarial es nueva; en su aplicación y medición. El objetivo principal es conocer el impacto del merchandising olfativo como estrategia sensorial. Se llevó a cabo una investigación de tipo exploratoria cuantitativa con análisis de datos descriptivos, consolidados a través de encuestas mediante muestreo aleatorio simple. Dentro de los principales resultados se evidencia que los aromas preferidos son las notas relajantes, para consumidores mayores de 36 años, seguidas de las notas cítricas, para consumidores menores de 35 años; en el caso de las ocupaciones, los empleados y empresarios prefieren aromas relajantes. Finalmente, los resultados mostraron aceptación y optimismo por parte de las personas hacia las marcas que desarrollan estrategias de merchandising olfativo.

Palabras clave: consumidor; marketing; merchandising olfativo; neuromarketing. JEL: D12; D87; M31; M37

\begin{abstract}
The smell perception has been related to human behavior; however as a business, strategy is relatively new, in its application and measurement, therefore the main objective of the article is to understand the impact of olfactory merchandising as a sensory strategy on consumers. Applied field research of quantitative exploratory type and under analysis of descriptive data was carried out, they were consolidated through surveys by simple random sampling. In the main results it is evident that the preferred aromas are the relaxing olfactory notes, for consumers over 36 years of age, followed by citrus notes, for consumers under the age of 35 years of age; in the case of occupations, employees and entrepreneurs prefer relaxing aromas. Finally, the results showed people's acceptance and optimism for the brands and products they develop olfactory merchandising strategies.
\end{abstract}

Keywords: consumer; marketing; merchandising olfactory; neuromarketing.

JEL: D12; D87; M31; M37 


\section{Resumo}

A percepção de cheiros sempre esteve relacionada ao comportamento do ser humano, porém, como estratégia de negócios é nova; na sua aplicação e medição. O objetivo principal é conhecer o impacto do merchandising olfativo como estratégia sensorial. Realizou-se uma pesquisa exploratória quantitativa com análise descritiva dos dados, estes foram consolidados por meio de inquéritos por amostragem aleatória simples. Dentre os principais resultados, fica evidente que os aromas preferidos são notas relaxantes, para consumidores com mais de 36 anos, seguidas de notas cítricas, para consumidores menores de 35 anos; no caso de ocupações, empregados e empregadores preferem aromas relaxantes. Por fim, os resultados mostraram aceitação e otimismo por parte das pessoas em relação às marcas que desenvolvem estratégias de merchandising olfativo.

Palavras-chave: consumidor; marketing; merchandising olfativa; neuromarketing.

JEL: D12; D87; M31; M37

\section{Introducción}

La presente investigación se refiere al tema de merchandising olfativo, que se puede definir como una técnica para crear vínculos emocionales entre la marca y el consumidor, a través de la memoria olfativa. La característica principal de este tipo de estrategia sensorial es que trabaja en relación directa con el cerebro, incrementando los recuerdos y las emociones simbólicas para el consumidor al momento de la compra (Alzate y Lujan, 2017; Grisales, 2019).

Todo este proceso empieza a verse contemplado a partir del siglo XVI, cuando el sentido de marca comercial comienza a ser utilizado con mayor frecuencia, dado que, cada persona tuvo que empezar a distinguir sus productos de los de sus colegas con alguna característica que lo identificara. Así, la identidad de las marcas actuales se ha ido construyendo a través del tiempo mediante una sutil e imperceptible acumulación de signos, mensajes y experiencias de distintas personas en la memoria del consumidor (Álvarez, 2011). 
A pesar de que en un comienzo se creía que los consumidores solo se veían influenciados por el precio y calidad del producto, el acto de compra no podía ni puede considerarse, como un acto puramente racional (De Garcillán, 2015).

Habría que decir también, que varios atributos de las tiendas y zonas de experiencia comunican mensajes simbólicamente a las personas que las visitan, por ejemplo, la apariencia, la ambientación y el merchandising, se proyectan y reflejan con la personalidad de los consumidores. Es así como los sentidos son el canal para proyectar la reputación de los puntos de venta (Martineau, 1958).

Así mismo, las empresas han tomado en cuenta todos estos factores sensoriales a la hora de diseñar sus marcas, productos y centro de experiencia, permitiendo la interacción directa con el cliente. Los interiores y exteriores de una tienda son espacios con una atmósfera de calidad, a los cuales se les debe desarrollar cuatro de las dimensiones controlables que Álvarez (2011) plantea dentro de su concepto de esquema atmosférico: visual, auditivo, olfativo y táctil. Estos elementos combinados influencian la conducta de los consumidores.

Las investigaciones de marketing sensorial comenzaron a tomar forma en los años 80 y principios de los años 90 del siglo pasado. Las compañías empezaron a invertir más para llegar a todos los sentidos de sus consumidores, teniendo en cuenta que para ellas ésta era la única forma de lograr la satisfacción del cliente y el reconocimiento de la marca/producto (Bonadeo, 2005).

Actualmente, se sigue hablando de la importancia para las empresas de llegar a los sentidos de los consumidores y crear experiencias memorables para ellos. Por esto, es importante resaltar una definición clara de lo que es el marketing sensorial, dado que tiene relación con los estímulos físicos y emocionales que los consumidores perciben a través de sus sentidos (Gómez y García, 2012). Es decir, todo lo que se utiliza para comunicarse a través de los sentidos y despertar en él, emociones y sensaciones a favor de la marca.

Así mismo, la tendencia del marketing sensorial está encaminada a maximizar la comunicación directa entre los sentidos y las emociones de las personas para crear estrategias efectivas de 
relacionamiento y recordación en los consumidores logrando el posicionamiento y la fidelización de las marcas.

En efecto, la importancia de establecer una influencia en el consumidor ha hecho que las empresas inviertan más tiempo en estudiar otras formas de relacionamiento subconsciente. Esto ha permitido que los sentidos tomen un papel muy importante, al punto que se han venido desarrollando estrategias como: en lo visual el logo símbolo, en lo auditivo el fonotipo, y en lo olfativo el odotipo (Grisales, 2019).

Relacionado con los odotipos, estos se definen como un estímulo olfativo que busca reforzar las marcas a través de un aroma especial, utilizando notas odoríficas que aportan a la personalidad y concepto de las marcas, para proyectarlo al público objetivo. El objetivo principal de los odotipos es ser un aroma inigualable para cada marca, y lograr una recordación positiva a un nivel consciente e inconsciente en las percepciones de los consumidores (Rodríguez, 2018).

Por lo tanto, se pueden asociar los estímulos olfativos como parte del marketing sensorial, siendo la estrategia que utiliza los olores para vincular emocionalmente al consumidor con las marcas y productos. Estos estímulos olfativos son utilizados por las marcas con el fin de despertar emociones y sentimientos influyendo directamente en el comportamiento del cliente, de tal forma que el olfato se pueda alterar, siendo uno de los sentidos más sensibles del ser humano; adicionalmente, dicho sentido se ha explotado comercialmente poco, cuando tiene la ventaja de que asocia rápidamente los productos y marcas, y además genera mayor permanencia en la memoria (Palacios, 2016).

De igual manera esta estrategia sensorial suscita nuevas emociones en las personas y su proceso es totalmente natural, puesto que no se está obligando ni tampoco afectando negativamente su permanencia ni su experiencia con la marca, al contrario, se generan sensaciones positivas para una mejor calidad de servicio. Para llevar a cabo esta estrategia de marketing sensorial olfativo, el cliente no tiene que realizar ningún esfuerzo, ya que naturalmente la persona está respirando, de tal manera que es como si se tratara por parte de la tienda o compañía de una especie de anuncio en el aire (López, 2017). 
Como nota importante para los puntos de venta, autores como Palacios (2016) aseguran que el marketing olfativo hace que los clientes prolonguen su estancia en dicho lugar, tal que la memoria pueda influir en las emociones provocando estados de ánimo que producen acciones de compra. En este mismo sentido, Díez (2013) plantea efectos sobre los consumidores cuando están expuestos a los aromas: (i) efecto de la percepción que el cliente asocia a características y desempeño de las marcas o productos, (ii) efecto de recuerdos como son la recuperación de contenidos y emociones, (iii) efecto de las emociones como sentimientos y que impacta la dopamina, (iv) efecto de la atención como es la focalización y concentración para la búsqueda de productos, (v) y por último el efecto del deseo tal que pueda catalizar el interés por la compra. El cliente asocia un aroma agradable con el producto, lo que crea una percepción positiva del mismo y por tanto aumenta las posibilidades de que vuelva a consumirlos o contratarlo (Hernández et al., 2016).

De la misma forma se pueden evidenciar otros conceptos y elementos relacionados directamente con el proceso de ambientación olfativa. Por ejemplo, la percepción es el primer proceso que fundamenta la reacción emocional conductual cuando los clientes están expuestos a un aroma, donde el autor Lersch (1966) considera que las sensaciones devienen a la persona desde el exterior y, para adquirir conciencia de ellas, dicha información debe ser captada mediante sentidos receptores, tales que luego pueda ser transmitida y transformada en una vivencia diaria; el sistema nervioso central transforma este conjunto de sensaciones provenientes de diversos canales sensoriales.

Como segundo proceso o elemento, se encuentran los receptores olfativos que son un tipo de neuronas que están siempre en contacto con el medio ambiente y al cual se encuentran expuestos, y desde donde reciben toda la información química olfativa. Las moléculas de olor están conectadas a receptores en las cilias, las cuales funcionan atrapando dichas moléculas en el aire que les rodea a través de los glomérulos (Alzate y Lujan, 2017). Como tercer elemento, la ambientación olfativa puede durar más tiempo en la memoria ya que el sistema olfativo y el sistema límbico tienen una proximidad biológica que permite que la sinapsis se transfiera rápidamente entre sus componentes (Krishna, 2012). 
Así es como el interés de las investigaciones por el sentido del olfato han venido fortaleciéndose; por ejemplo, en el año 2014 se estudiaron a 26 personas que percibieron más de 128 moléculas odorantes y realizaron más de 264 comparaciones con aromas desconocidos para evadir cualquier sesgo. Con esto, se logró revelar que una persona en promedio puede detectar entre al menos 1 billón de aromas y que estas mismas personas son capaces de distinguir aromas unos de otros (Bushdid et al., 2014), dando así un giro a las estrategias de marketing tradicionales, para continuar con la era de las sensaciones, emociones y experiencias a través de los sentidos, en este caso, el olfato.

Definitivamente el aroma puede resultar eficaz para funcionar como una estrategia sensorial que ayude a promover el posicionamiento de las marcas; es necesario que las marcas elijan aromas estables en el tiempo, que actúen como parte de su identidad corporativa e imagen, asociado a elementos fáciles de percibir por el mercado objetivo. Crear una percepción odorífica que perdure en el tiempo, es la denominada desarrollo de odotipo, el cual es tomado como un atributo indicador a la identidad y personalidad de la marca, que se le utiliza para identificar un producto o servicio más allá de sus aspectos visuales (Bonadeo, 2005).

Adicionalmente, las personas reconocen aromas predeterminados debido a que cada uno es asociado a productos como los perfumes, esencias, ambientadores, entre otros. No obstante, aunque algunas organizaciones hoy en día utilizan aromas en sus puntos de venta, no todos van con relación a sus conceptos de marca, lo que hace que habitualmente el merchandising olfativo no sea considerado como una estrategia sensorial que modifica y altera el comportamiento. La ausencia de cuestionamientos y estudios sobre la conducta y la relación directa con el aroma no ha permitido que las marcas y productos lo utilicen de manera relevante y eficaz (Bonadeo, 2005).

Avanzando en la importancia del tema de la percepción olfativa, el olor juega un papel primordial, no obstante, esta percepción es netamente individual formada por la esencia del individuo que la vivencia, es por esta razón que las empresas deben tener en cuenta dicha información en el marketing olfativo, que quizás no podrán satisfacer de manera colectiva a sus públicos en lo que al aroma se refiere (Grisales, 2019). 
Dicho lo anterior, el sistema límbico está estrechamente relacionado con las emociones y se encuentra ligado a la percepción del olor, es por esto, que cuando estamos expuestos a un aroma, se intensifican las respuestas emocionales, focalizando y activando el comportamiento del consumidor (Grisales, 2019).

Para ilustrar la unión del sistema límbico, aromas y emociones, Lindstrom (2005) realizó un estudio vinculando la vivencia de los consumidores con marcas a un nivel emocional, considerando los cincos sentidos en el acto de compra, de tal forma que pudiese analizar si la respuesta emocional dominaba el pensamiento racional.

De igual modo un estudio realizado por Keller (2014) autora principal de la investigación de la Universidad de Rockefeller de Nueva York, menciona que los consumidores son capaces de reconocer y distinguir entre un billón de olores diferentes, y también se confirmó que el sentido del olfato está estrechamente relacionado con el comportamiento humano. La felicidad de los consumidores está relacionada con la activación de uno o varios sentidos los cuales se activan positivamente cuando las estrategias vinculan los canales multisensoriales, dando un equivalente que entre más percepción sensorial mayor recuerdo y fidelidad (Álvarez, 2011).

Además, los estudios de neuromarketing, permiten profundizar en la efectividad del marketing olfativo pues se vincula el uso del cerebro en la toma de decisiones durante el proceso de compra. En el año 2005 se realizó un escáner mental a 2.081 consumidores que fueron sometidos a estímulos publicitarios visuales, olfativos y auditivos, mientras se controlaba su actividad cerebral, dilatación de la pupila, actividad sudorípara y movimientos faciales. Una de las conclusiones más importantes fue que el $83 \%$ de la publicidad de hoy en día, solo capta uno de los sentidos: la vista. Ahora bien, los resultados evidencian que, al usar los cinco sentidos, el consumidor podía memorizar con mayor facilidad la marca, tal que el marketing sensorial podría lograr mejores resultados en los procesos de compra de los individuos (Lindstrom, 2005).

Según Harrop, fundador de la agencia Brand Sense, hay un exceso de confianza por parte de las organizaciones en la utilización de la comunicación publicitaria a través del sentido de la vista: “El $83 \%$ de las campañas se centran en los ojos y solo el $4 \%$ de recursos de del marketing se 
dirigen al olfato, el gusto y el tacto" (De Garcillán, 2015, p. 468). Esto puede suceder por desconocimiento de los empresarios y las agencias de marketing que prefieren concentrarse en lo seguro y lo comprobado, así mismo se puede evidenciar que la vinculación de otros sentidos es un proceso más costoso que requiere una nueva curva de aprendizaje y vinculación de innovación, creatividad y tecnología.

El proceso es sencillo, las sensaciones percibidas a través de los cinco sentidos activan directamente el sistema neuronal y generan emociones. Cuanto más intensas, más se quedan en la memoria, y si ese recuerdo o experiencia está relacionada con momentos positivos en la vida de la persona esto se refleja en la actitud hacia la marca, lo cual es perfecto. Por esto, la publicidad del futuro deberá estar basada en el subconsciente y no el consciente.

Cuando se habla de merchandising olfativo se puede decir que son reacciones positivas del consumidor, frente un aroma expuesto que puede despertar un estado de ánimo ya sea positivo o negativo de acuerdo con su memoria episódica que le permitirá confirmar si se trata de un aroma pasado o si es uno totalmente nuevo para codificar (Alzate y Lujan, 2017).

En síntesis, la relación entre el aroma y la marca no es al azar, ya que el primero se debe verificar la personalidad de marca, y el sector al cual pertenece la categoría de productos o servicios que ofrece al mercado. De esta forma descubrir la nota olfativa que promueva el concepto de la marca (Grisales, 2019).

El merchandising olfativo tiene diferentes aplicaciones como lo menciona Gómez (2017), el primero, aroma vendedor; es el aroma que se utiliza para atraer la atención de los productos como táctica en una estrategia promocional. El segundo, olor de producto, estos son aromas específicos que hacen parte de este desde su esencia y creación. El tercero, olor ambiental, es aquí donde vemos aplicada la estrategia de odotipos y el estudio de notas olfativas las cuales son expuestas en los puntos de venta por medio de tecnología, unidades olfativas o de manera manual a través de spray, brindándole valor sensorial a la marca, generando reconocimiento por medio de un aroma que la identifica. 
Por lo cual esta investigación se realizó con el fin de descubrir la percepción que tienen los consumidores sobre el desarrollo e implementación de aromas como estrategia sensorial para las marcas y/o productos en la ciudad de Cali. Los cuales utilizan aromas creados o específicos como estrategias comerciales para incentivar sus ventas y relacionamiento con todos sus públicos.

Para ello, se propusieron tres objetivos fundamentales. El primero es conocer la percepción de los consumidores sobre las notas olfativas; el segundo es validar la nota olfativa de preferencia entre las familias odoríficas: dulces, cítricas y relajantes; y el tercero es proponer el conocimiento del merchandising olfativo como estrategia sensorial para las empresas ubicadas en la ciudad de Cali.

Para cumplir con los objetivos del artículo, se llevó a cabo una investigación aplicada en campo, del tipo exploratoria cuantitativa teniendo en cuenta que a nivel empírico en Colombia no hay suficiente información cuantitativa del marketing olfativo y su respectiva validación, llegando a ser esta investigación de las primeras en su área. Con esto último, también se pretende que esta investigación, tanto los análisis del marco de referencias y de datos empíricos, sirvan de referencia para futuras investigaciones, tanto académicas y científicas en el área del marketing sensorial.

Se pretende generar resultados a partir de una investigación cuantitativa que permita conocer la percepción que tienen los consumidores frente a los de aromas utilizados como estrategia de merchandising olfativo, de tal manera que estos hallazgos generen nuevo conocimiento que pueda ser utilizado por las empresas para mejorar, la implementación y evaluación de sus estrategias sensoriales.

El artículo se divide en cinco secciones; en primer lugar, se encuentra esta introducción. En segundo lugar, se presenta el marco teórico y de referencias. En la tercera sección se presentan los materiales y métodos empleados para lograr los objetivos. Luego se publican los resultados de las encuestas y se hacen las respectivas discusiones. Finalmente se encuentran las conclusiones.

\section{Metodología}

Para cumplir con los objetivos de la investigación, se llevó a cabo una investigación aplicada en campo, del tipo exploratoria cuantitativa teniendo en cuenta que a nivel empírico en Colombia no 
hay suficiente información cuantitativa del marketing olfativo y su respectiva validación. El muestreo fue probabilístico aleatorio simple.

El diseño de la muestra para esta investigación fue constituido por un universo poblacional integrado por hombres y mujeres, mayores de 18 años y menores de 60 años, que vivan en la ciudad de Cali, en zonas norte, sur y oriente. La muestra está constituida por personas activas, dinámicas y con disposición al test de percepción, con un estilo de vida profesional, empresario, emprendedor, estudiantil u hogareño. Estos perfiles son relevantes en el estudio ya que aportan a los componentes comportamentales que se crean en el desarrollo de la investigación. La muestra también debe ser apta para percibir aromas. En este sentido, dado que la población es superior a 300.000 individuos $^{1}$, la fórmula para el cálculo de la muestra es la siguiente:

$$
n=\frac{Z^{2} * P *(1-P)}{e^{2}}
$$

Donde $Z$ es el valor de la distribución normal estándar, $\mathrm{P}$ es la participación de la población que contiene la característica, (1- P) la participación de la población que no la contiene y $e$ es el error permitido. En este sentido, para un nivel de confianza del $95 \%$ el $Z$ calculado es 1,96. Este valor se encuentra en la tabla de la distribución normal estándar. El nivel de confianza indica la probabilidad de que los resultados de la investigación sean ciertos. Lo anterior lleva a pensar en que el error permitido es del $5 \%$, luego $e=0,05 . P=65 \%$ y por lo tanto $1-P=35 \%$, este es un valor que se basa en el supuesto de la que la mayoría de las personas compran productos teniendo en cuenta el sentido olfativo ${ }^{2}$. Por lo cual el tamaño de muestra es:

$$
n=\frac{1,96^{2} * 0,65 * 0,35}{0,05^{2}}=350
$$

\footnotetext{
${ }^{1}$ Superior a este valor se puede considerar la población como infinita y por lo tanto basta con usar la fórmula para un tamaño de población infinita o cuando ésta no se conoce.

${ }^{2}$ Como en Colombia las investigaciones a nivel empírico del marketing olfativo son más bien recientes, es de aceptar que esta proporción no es un valor definido completamente, razón por la cual más bien es un supuesto en este caso. Queda abierto para próximos trabajos investigar proporciones del marketing sensorial en los consumidores, que den soporte empírico a este tipo de trabajos.
} 
Por lo tanto, la muestra debe ser de 350 personas como mínimo.

Respecto al instrumento para recolección de la información se usó un cuestionario de 8 preguntas, algunas de única respuesta y otras de respuesta abierta con el fin de leer la opinión de los encuestados. La Tabla 1 muestra las 8 preguntas que se hicieron a los participantes, de donde se discriminan las preguntas por objetivo específico y componentes comportamentales de los consumidores: percepción, emoción, sentidos y de asociación. Esta tabla se desarrolla para vincular el proceso de categorización realizado con la información dando respuesta a los objetivos de investigación y ubicando estratégicamente cada componente, de una manera más sintetizada y organizada, permitiendo un mejor análisis del documento ${ }^{3}$.

\section{Tabla 1}

Categorización de preguntas del cuestionario

\begin{tabular}{|c|c|c|}
\hline $\begin{array}{l}\text { Objetivo } \\
\text { específico }\end{array}$ & Componente & Pregunta \\
\hline $\begin{array}{l}\text { Conocer la } \\
\text { percepción de las } \\
\text { personas para las }\end{array}$ & Perceptivo & $\begin{array}{l}\text { 1. ¿Cuál diría usted que es el nivel de preferencia para } \\
\text { cada una de las notas olfativas? Evalúe su nivel de } \\
\text { preferencia organizándolos en una escala de } 1 \text { a } 7 \text {. }\end{array}$ \\
\hline $\begin{array}{l}\text { notas olfativas } \\
\text { percibidas }\end{array}$ & Emocional & $\begin{array}{l}\text { 2. ¿Qué sensación o emoción le genera el aroma } \\
\text { seleccionado? }\end{array}$ \\
\hline \multirow{3}{*}{$\begin{array}{l}\text { Validar la nota } \\
\text { olfativa de } \\
\text { preferencia entre } \\
\text { la categoría de } \\
\text { aromas dulces, } \\
\text { cítricas y } \\
\text { relajantes }\end{array}$} & Cognitivo & $\begin{array}{l}\text { 3. La nota olfativa calificada con mayor nivel de } \\
\text { preferencia ¿por qué es su primera opción? }\end{array}$ \\
\hline & Asociación & $\begin{array}{l}\text { 4. ¿El aroma seleccionado le recuerda un espacio, } \\
\text { producto o una marca en específico? }\end{array}$ \\
\hline & Razonamiento & $\begin{array}{l}\text { 5. ¿Qué espacios o productos le gustaría ambientar con } \\
\text { este aroma? }\end{array}$ \\
\hline $\begin{array}{l}\text { Proponer el } \\
\text { conocimiento del } \\
\text { merchandising } \\
\text { olfativo como } \\
\text { estrategia } \\
\text { sensorial en la } \\
\text { ciudad de Cali }\end{array}$ & Sentidos & $\begin{array}{l}\text { 6. ¿Qué sentido se estimula más en usted al momento de } \\
\text { comprar un producto o servicio? } \\
\text { 7. ¿Cree usted que las marcas y/o productos deberían } \\
\text { tener un aroma que los identifique? } \\
\text { 8. ¿Cómo describiría la estrategia de marketing olfativo } \\
\text { que consiste en ambientar la marca, sus productos y el } \\
\text { punto de venta con un aroma específico? }\end{array}$ \\
\hline
\end{tabular}

Fuente: autoría propia.

\footnotetext{
${ }^{3} \mathrm{Si}$ el lector desea acceder a la ficha técnica del instrumento debe enviar un correo electrónico a los autores para que en respuesta le sea enviada.
} 


\section{Resultados}

En la encuesta realizada se tuvo en cuenta tres variables adicionales para cada consumidor, además de las preguntas mencionadas en la Tabla 1; dichas variables son: género, ocupación del individuo $\mathrm{y}$ edad. Esto permite segmentar los futuros mercados para las notas olfativas y por lo tanto para las empresas que tomen decisiones estratégicas para incrementar sus ventas, teniendo en cuenta el merchandising olfativo.

Es así como en la Tabla 2 se logra observar que el 64\% de los encuestados fueron mujeres y el $36 \%$ fueron hombres. Así mismo, el 58\% de las personas fueron empleados o empresarios. Relacionado con la variable de edad, la proporción de encuestados más alta estuvo en el rango de 18 a 25 años, seguido por el rango de 26 a 35 años y así sucesivamente fue cayendo la proporción por rangos hasta llegar a los 60 años de edad.

\section{Tabla 2}

Distribución de la muestra por ocupación y rangos de edad controlando por género

\begin{tabular}{clccc} 
& & Femenino & Masculino & Total \\
\cline { 3 - 5 } & Empleado/a & $18 \%$ & $21 \%$ & $39 \%$ \\
Ocupación & Empresario/a & $13 \%$ & $6 \%$ & $19 \%$ \\
actual & Estudiante & $14 \%$ & $8 \%$ & $23 \%$ \\
& Hombre o mujer de hogar & $18 \%$ & $1 \%$ & $20 \%$ \\
\cline { 2 - 5 } & \multicolumn{1}{c}{ TOTAL } & $64 \%$ & $36 \%$ & $100 \%$ \\
\hline \multirow{2}{*}{ Rangos de } & $18-25$ & $16 \%$ & $9 \%$ & $26 \%$ \\
edad & $26-35$ & $17 \%$ & $9 \%$ & $25 \%$ \\
& $36-45$ & $15 \%$ & $8 \%$ & $23 \%$ \\
& $46-55$ & $9 \%$ & $8 \%$ & $17 \%$ \\
\cline { 2 - 5 } & $56-60$ & $7 \%$ & $3 \%$ & $9 \%$ \\
\hline
\end{tabular}

Fuente: cálculos propios.

Estos resultados son interesantes en la medida que aquellos que tienen un poder adquisitivo mayor en la encuesta, como son empleados y empresarios, son precisamente los que pueden comprar o decidir sobre las compras de productos con aromas que ambienten el lugar de trabajo, el lugar donde viven u otras áreas. También, que la mayoría de encuestados se encuentren en los rangos de 
edad más jóvenes, puede generar estrategias de mercadeo sobre notas olfativas para una población que tendrá por mucho tiempo capacidad de compra y fidelización al producto y a la marca.

Especialmente relacionado con las preguntas de la Tabla 1, en adelante se hará la interpretación de las respuestas por parte de los encuestados. Es así como, por ejemplo, la Figura 1 reúne en tres paneles $(\mathrm{A}, \mathrm{B}, \mathrm{C})$ el cruce de variables y respuestas para la pregunta número 1. La primera pregunta hace referencia al nivel de preferencia que el consumidor tiene sobre las tres notas olfativas en estudio.

\section{Figura 1}

Nivel de preferencia para cada una de las notas olfativas

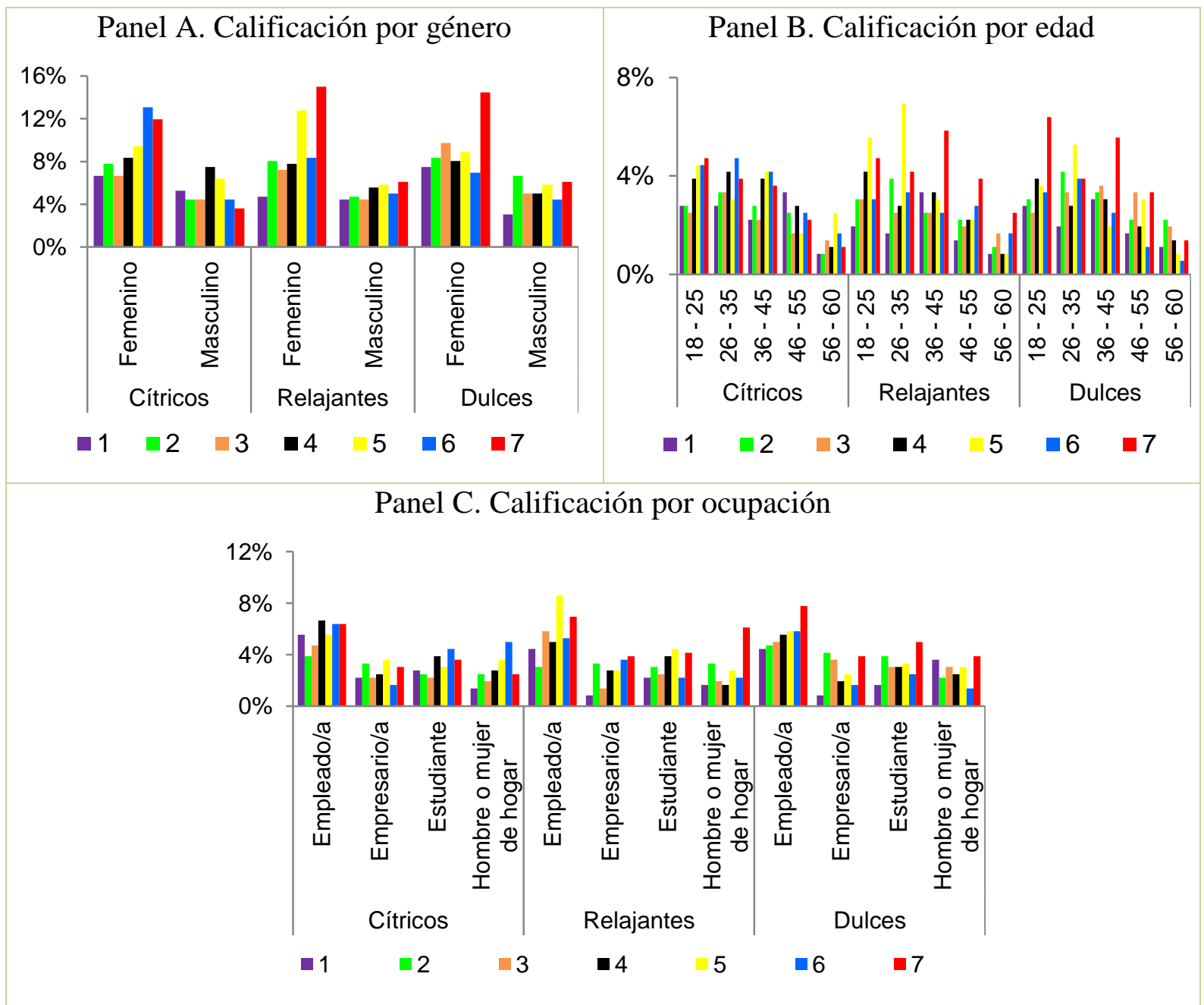

Fuente: cálculos propios. 
De esta forma, teniendo en cuenta que las calificaciones 5, 6 y 7 implican mayor aceptación del aroma, entonces se puede observar en la Figura 1 Panel A que los aromas relajantes son los de mayor preferencia para las mujeres (36\%), seguidos muy de cerca por los cítricos (34\%) y dulces (30\%). Respecto a los hombres, la mayor preferencia son los aromas relajantes (17\%), seguidos por los aromas dulces (16\%) y cítricos (14\%).

Respecto a las preferencias de aromas por rangos de edad, en el Panel B de la Figura 1 se puede observar que las notas olfativas cítricas son menos preferidas por los consumidores a medida que suben los rangos de edad, es decir, ellas son más preferidas por las personas más jóvenes. Por el lado de las notas olfativas relajantes, éstas tienen mayor aceptación (calificación 7) por parte de las personas en rangos de edad mayores, por encima de los 36 años de edad, mientras que los rangos de 18 a 35, dan una calificación de 5. En cuanto a las notas olfativas dulces, la mayor preferencia es para los individuos con menos de 45 años de edad, mientras que para los mayores estas notas olfativas dulces no tienen mayor aceptación.

Teniendo en cuenta la ocupación, se puede analizar en el Panel C, que las mayores preferencias (calificaciones de 5 a 7) hacia los aromas relajantes son por parte de los empleados y empresarios, los aromas cítricos y relajantes para las amas de casa y hombres que permanecen en el hogar, mientras que para los estudiantes no hay preferencia significativa en alguna de las tres notas olfativas.

La sensación que le genera al encuestado la nota olfativa de mayor preferencia, la Figura 2 en los paneles A y B permiten ver los resultados. Es así como, por ejemplo, en el Panel A, del 100\% de los encuestados, el $47 \%$ reportaron felicidad, el $27 \%$ tranquilidad y el $26 \%$ otro tipo de sensación. De aquí, es importante resaltar que el 28\% resultaron ser mujeres en edad de 18 a 45 años a las cuales el aroma seleccionado les reportaba felicidad. 


\section{Figura 2}

Sensación que le genera el aroma seleccionado
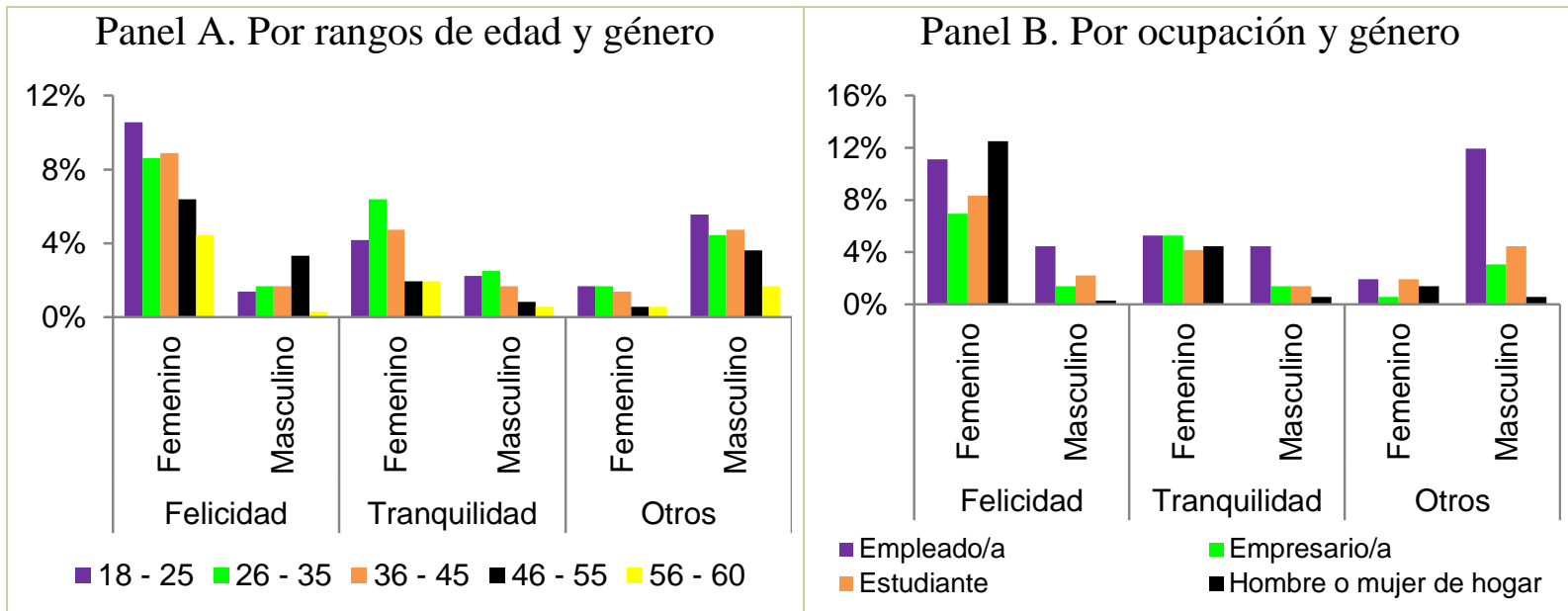

Fuente: cálculos propios.

En cuanto a la ocupación, en el Panel B, se puede notar que la mayor sensación es de felicidad para las mujeres empleadas y amas de casa. Por el contrario, para los hombres empleados no hay una sensación específica a partir del aroma seleccionado. En cuanto a los empresarios y estudiantes, la mayor sensación también es la de felicidad.

En seguida se analizan estadísticas descriptivas con el fin de validar la nota olfativa de preferencia, en primer lugar, respecto al por qué dicha calificación, luego sí dicho aroma de preferencia le recuerda algún espacio, productos, tiempo o marca en particular, y en tercer lugar los espacios que le gustaría al consumidor ambientar con la nota olfativa de mayor ponderación.

Respecto a la pregunta 3, que se relaciona con la justificación de la calificación más alta a la nota olfativa de preferencia, debido a que fue una pregunta abierta al consumidor, se decidió unificar las respuestas en tres categorías: (i) aquellas respuestas que el consumidor asoció a características positivas del aroma, como por ejemplo suave, sensual, agradable, entre otras; (ii) aquellas respuestas asociadas a reacciones positivas como recuerdos, identificación con algo o alguien, estadía en algún lugar como el campo, entre otras; (iii) percepción individual o propia como por ejemplo que el aroma lo percibía fuerte, o como olor a chicle, o porque tan solo le parecía que dicha nota olfativa era el mejor aroma, o que dicha nota era nueva para él, entre otras percepciones. 
Dicho lo anterior, en la Figura 3 se puede ver que la mayoría de encuestados dieron la calificación más alta a la nota olfativa por sus características positivas, es decir, por los aromas como dulce, sensual, equilibrado, etc. El $44 \%$ fueron mujeres que eligieron la nota olfativa por sus características positivas; de ese 44\%, el 32\% estuvieron entre los 18 y 45 años de edad. En cuanto a la ocupación, en su mayoría las empleadas y amas de casa fueron quienes derivaron sentimientos positivos de las notas olfativas seleccionadas.

\section{Figura 3}

La nota olfativa calificada con mayor nivel de preferencia, ¿por qué es la primera opción?

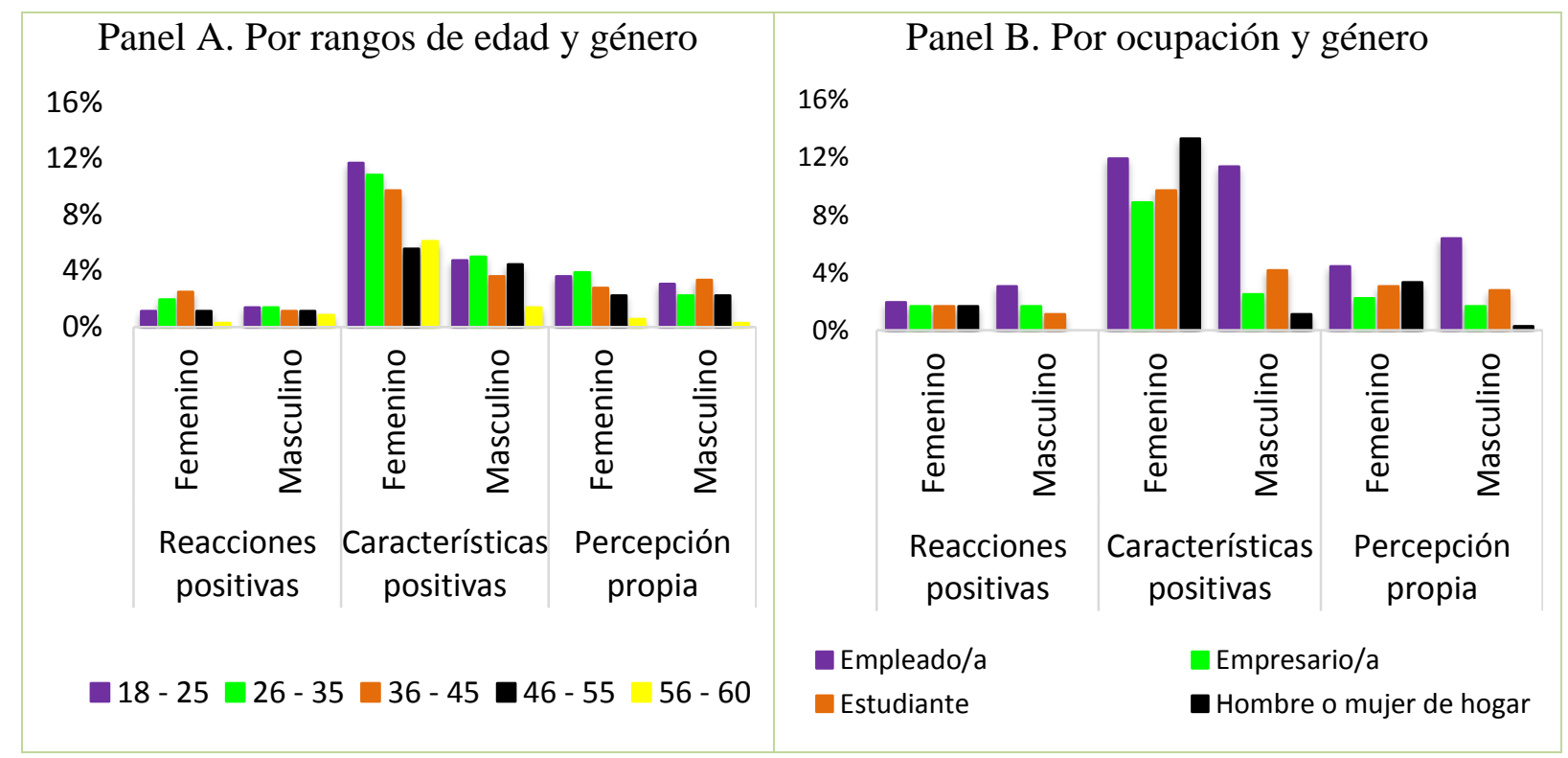

Fuente: cálculos propios.

En cuanto a si el aroma de preferencia le recuerda o no un espacio, productos, tiempo o una marca en específico, en la Figura 4 se puede notar que a la mayoría de encuestados la nota olfativa de preferencia si le trae recuerdos, mayormente a las mujeres que a los hombres. En cuanto a la edad, son las mujeres menores de 45 años las que tienen esta clase de sentimientos, y por ocupación, son los empleados (hombres y mujeres) quienes se afectan más en sus recuerdos por el aroma seleccionado. 


\section{Figura 4}

El aroma seleccionado le recuerda un espacio o una marca en específico

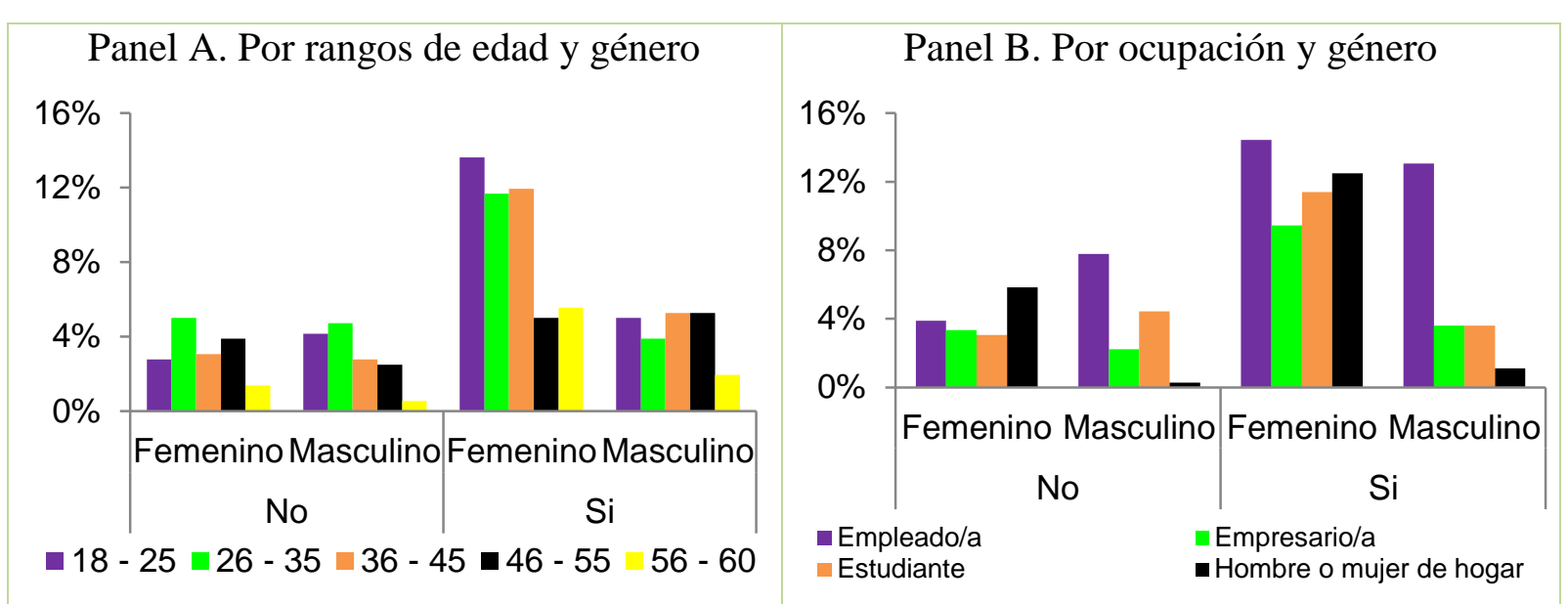

Fuente: cálculos propios.

Por su parte la Figura 5 muestra las categorías de espacios, productos, tiempos o marcas que recuerdan las personas por el aroma de la nota olfativa seleccionada. Por ejemplo, los lugares más recordados por los encuestados son los centros comerciales con $29 \%$ de aceptación, seguido de dulces y bebidas con $22 \%$ y en el tercer puesto el campo o lugares asociados con la naturaleza $(13 \%)$.

\section{Figura 5}

Categorías de espacios, productos, tiempos o marcas

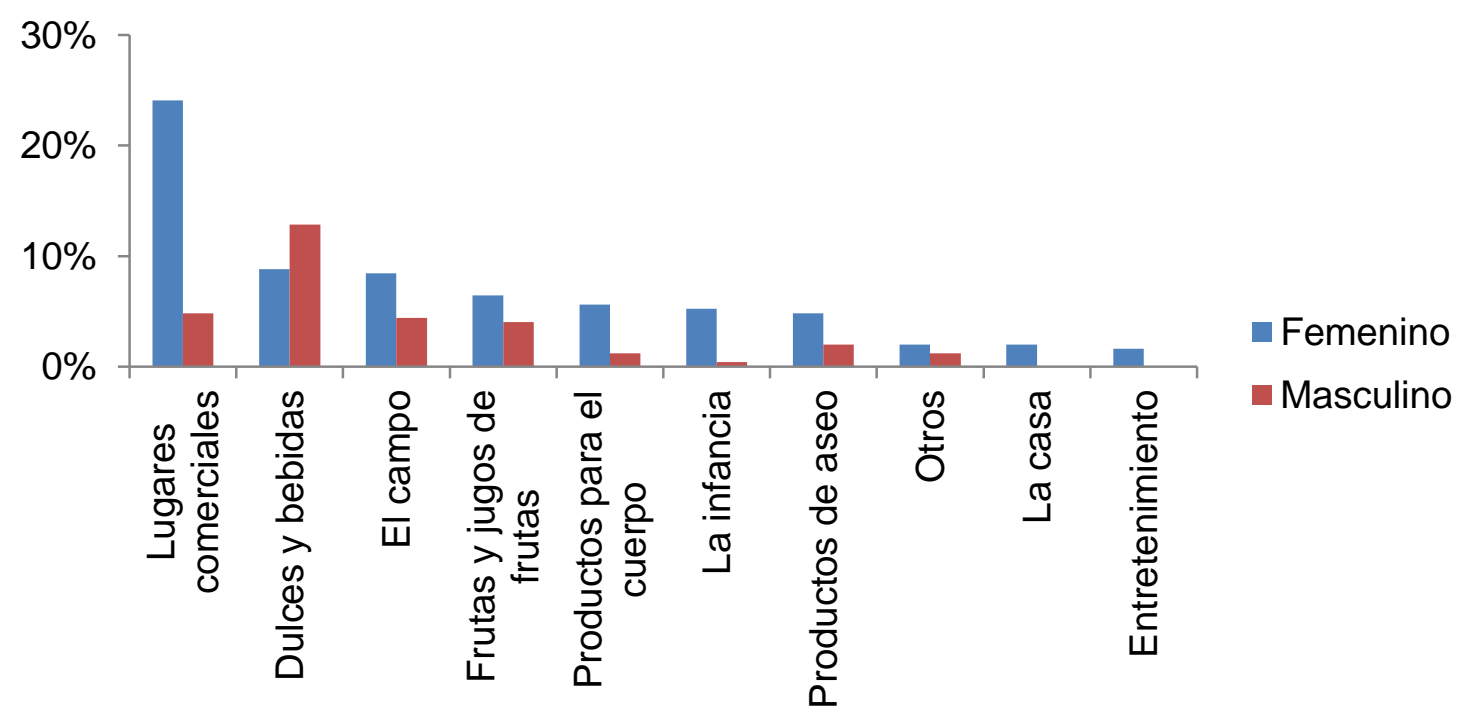

Fuente: cálculos propios. 
De otro lado, se puede deducir información sobre los espacios o productos que al consumidor le gustaría ambientar con el aroma seleccionado. Así, el 22\% de los encuestados respondieron que el lugar donde viven como casa o apartamento (Apto) es el de mayor preferencia por ambientar, sin discriminar el espacio dentro de dicho lugar, mientras que el $47 \%$ fueron específicos con el espacio por ambientar dentro del lugar donde viven, discriminados con el $14 \%$ en la sala, el $14 \%$ en los baños, el $13 \%$ en la habitación y el $6 \%$ cajones y ropa.

En términos generales, el 69\% de los encuestados estarían dispuestos a darle aroma a los espacios del apartamento o casa con la nota olfativa seleccionada, que relacionado con las respuestas anteriores, la mayoría de personas desearían ambientar sus hogares con aromas relajantes, seguidos por aromas cítricos. Este resultado es interesante, en la medida que, por ejemplo, para los individuos que laboran en empresa, al regresar a sus casas, pudieran sentir a través del olfato la sensación de descanso y relajación en sus hogares; y en términos generales, para todos aquellos individuos que llevan a cabo actividades fuera de su lugar de vivienda.

\section{Figura 6}

Espacios o productos que le gustaría ambientar con el aroma de su preferencia

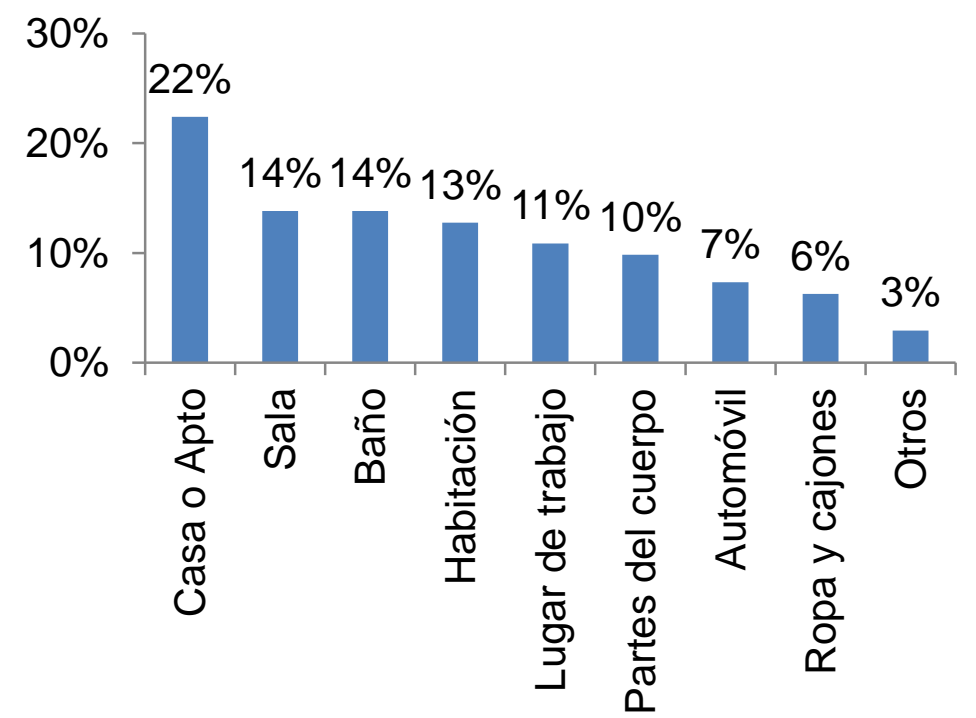

Fuente: cálculos propios. 
Relacionado con el tercer objetivo de la investigación que buscar extraer información sobre la relevancia del merchandising olfativo como estrategia sensorial de ventas, la Figura 7 permite ver que del $100 \%$ de los encuestados, el $49 \%$ compra productos a partir de la vista, seguido por el $42 \%$ de compras a partir del olfato, resultado más que interesante para incrementar las ventas de las empresas bajo una estrategia adecuada del merchandising olfativo.

\section{Figura 7}

Sentido que más se estimula al momento de comprar

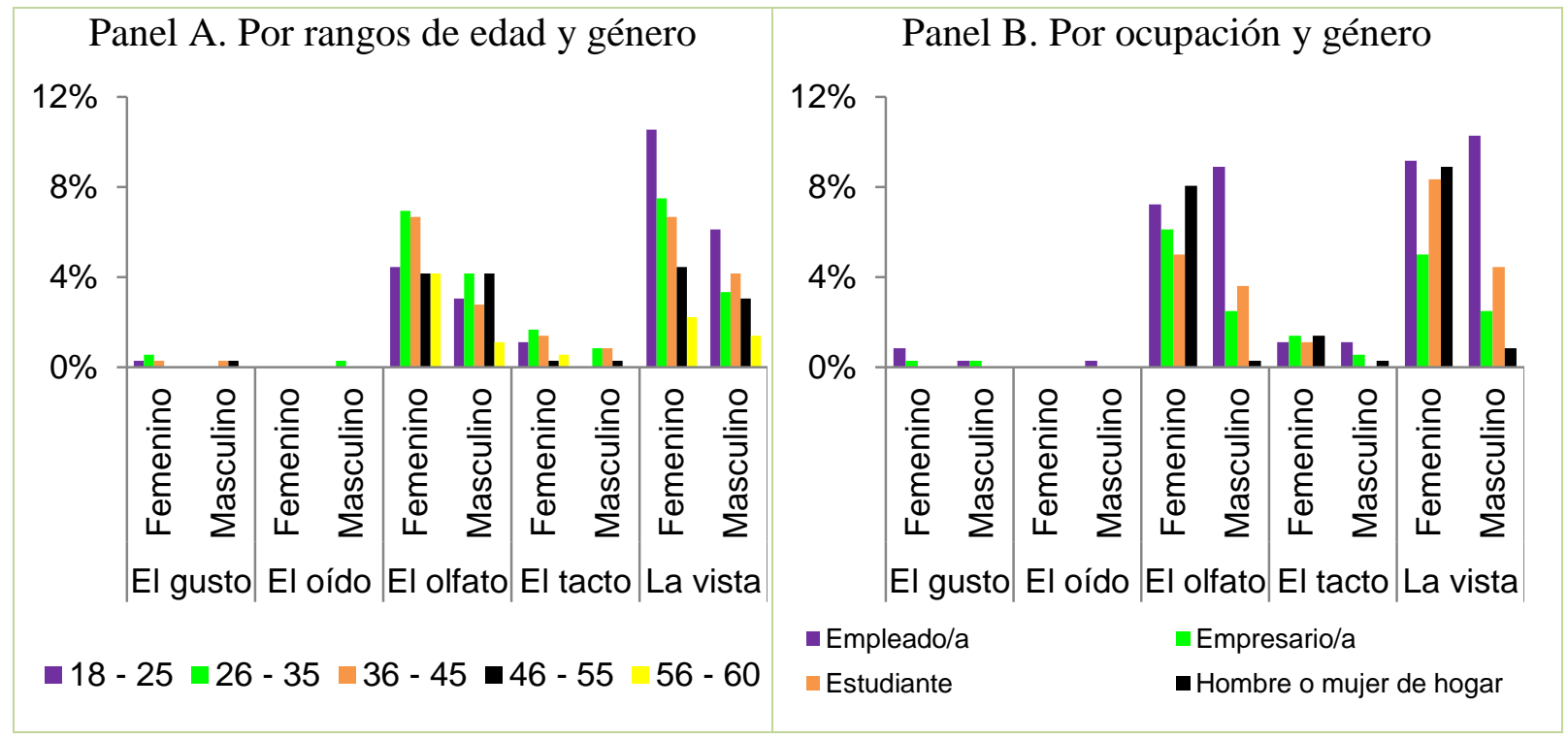

Fuente: cálculos propios.

Relacionado con el sentido del olfato, del $42 \%$ de compras influenciadas por los aromas el $26 \%$ son hechas por las mujeres, de las cuales la mayoría están entre 26 y 45 años de edad (14\%). Con relación a las ocupaciones, son los empleados -hombres y mujeres - (16\%) y amas de casa (8\%) los que más compran influenciados por notas olfativas.

Cuando se indagó a los encuestados sobre si las marcas o productos deberían tener un aroma de identificación (Figura 8), la gran mayoría respondieron afirmativamente (89\%), de los cuales el $58 \%$ fueron mujeres y el $31 \%$ hombres, el $66 \%$ personas entre 18 y 45 años de edad, el 34\% empleados en empresas (hombres y mujeres) y el $17 \%$ mujeres amas de casa. 


\section{Figura 8}

¿Las marcas y/o productos deberían tener un aroma que los identifique?

Panel A. Por rangos de edad y género

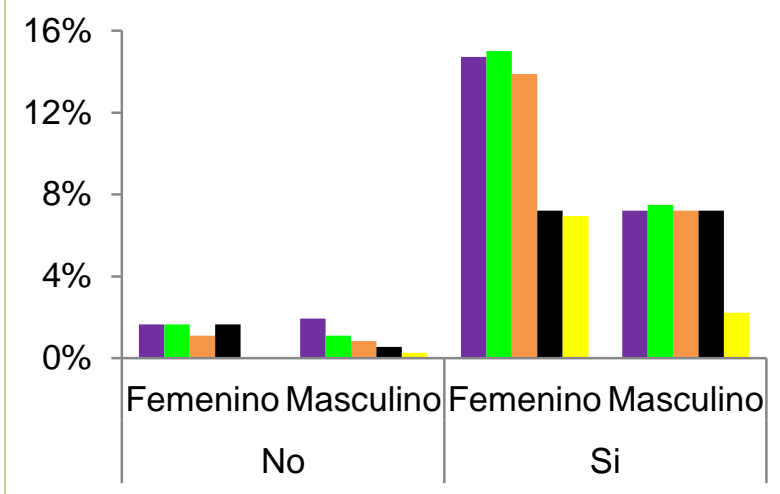

$\square 18-25 \square 26-35 \square 36-45-46-55 \square 56-60$
Panel B. Por ocupación y género

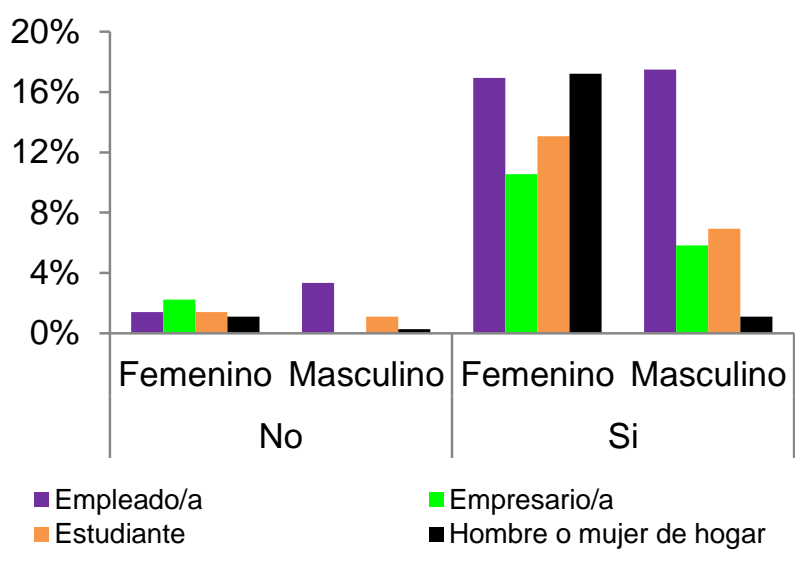

Fuente: cálculos propios.

Finalmente, ante la pregunta por la descripción que el encuestado le daría a la estrategia de marketing olfativo teniendo en cuenta que dicha estrategia consiste en ambientar los productos y el punto de venta, según la Figura 9, el 51\% considera que es inolvidable y el $41 \%$ que es novedosa, mientras que el restante $7 \%$ la considera como una estrategia de ventas normal, que se podría interpretar como igual a las demás estrategias de marketing. Así mismo, en términos generales, presentan mayor interés por dicha estrategia las personas menores de 45 años, o de otra forma, a medida que van aumentando los años de edad, disminuye el interés por el marketing olfativo.

Con relación a las ocupaciones, la estrategia del marketing olfativo es novedosa para el 18\% de los empleados e inolvidable para el 17\%, seguido en un $11 \%$ como inolvidable para las mujeres amas de casa. En este sentido, el 46\% de los encuestados según la ocupación de empleados y amas de casa, tienen una actitud favorable para el uso del marketing como estrategia de ventas, lo cual es interesante considerando que son ocupaciones determinantes por el lado de la demanda de productos. Por su parte, y aunque la ocupación de empresarios (por el lado de la oferta de productos) solo tiene una opinión favorable (inolvidable y novedosa) del 18\% hacia el marketing olfativo, es de lejos superior al $1 \%$ que tan solo opina que es una estrategia normal. 


\section{Figura 9}

Descripción del marketing olfativo como estrategia de venta
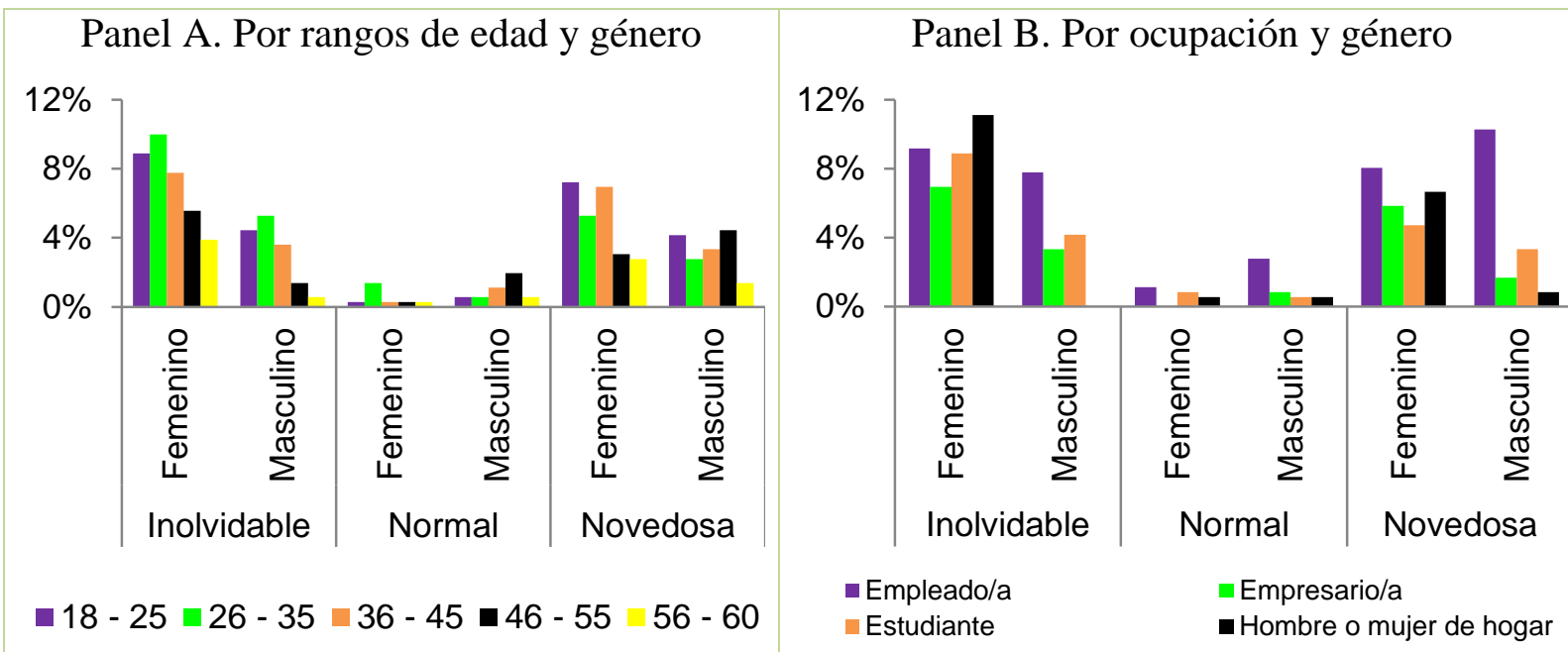

Fuente: cálculos propios.

\section{Conclusiones}

Con relación al objetivo de conocer la percepción de los consumidores sobre las notas olfativas, habiendo hecho los análisis de las Figuras 1 y 2, se puede concluir que la nota olfativa con mayor aceptación; son las relajantes, tanto para mujeres como para hombres mayores de 36 años. Los aromas relajantes están relacionados con salud y la paz interior, para los menores de 35 años, las notas olfativas preferidas son las cítricas. Las notas cítricas son muy fragantes, identificándose de mejor manera con los consumidores jóvenes y modernos en sus procesos de consumo. Por otra parte, los aromas relajantes son preferidos por empleados y empresarios. Ya que son estas personas las que tienen mayor carga laboral y siempre están buscando liberar su estrés y estar en un buen estado anímico para seguir en sus actividades.

En cuanto al componente emocional, la mayoría reportó felicidad, lo cual, combinado con lo antes dicho, indica que la nota olfativa relajante tiene sensaciones de felicidad sobre los consumidores en la ciudad de Cali, y mayormente para las mujeres de edad entre 18 y 45 años. Un estudio realizado por Alzate y Lujan (2017) en la ciudad de Medellín confirma que las consumidoras de almacenes vestuario y accesorios, tienen sensaciones de felicidad al ingresar a un almacén con aromas cítricos y relajantes. Los aromas producen emociones y esas emociones van ligadas a las 
experiencias que estén viviendo los consumidores en el momento y también dependiendo de su personalidad, ya que los aromas son percibidos de manera individual no colectiva (Grisales, 2019).

Por su parte el objetivo de validar la nota olfativa de mayor preferencia, se alcanzó con los datos presentados en las Figuras 3, 4, 5 y 6, de donde se concluye que la mayoría de personas aceptan las notas olfativas por sus características positivas como olor dulce, sensual, entre otros adjetivos, Así mismo, dichas características positivas activan la memoria episódica de los consumidores (Bonadeo, 2005). Es por esta razón que un visitante puede generar motivación al ingresar a una tienda o puede preferir no ingresar según la información que tenga del aroma que percibe en ese instante. Las notas utilizadas en marketing olfativo, no deben estar relacionados directamente con aromas muy expuestas en el ambiente comercial, ya que no cumplirán su objetivo de codificarse como un aroma único relacionado con dicha marca. En este sentido, el empresario debe tener en cuenta que en cada odotipo creado debe existir una estrategia genuina que denote un aroma único vinculado con la personalidad de la marca y su concepto sensorial para el mercado objetivo.

Adicionalmente, la mayoría de encuestados estarían dispuestos a aromatizar los espacios de su hogar con aromas relajantes, resultado por demás interesante, dado que para individuos que realizan actividades fuera del hogar, como empleados, empresarios y estudiantes, una vez regresan a su vivienda, los aromas relajantes podrían servir para hacer mucho más amigables sus espacios personales y familiares, que sin duda aportaría a la calidad de vida de las personas. De este tipo de comportamientos donde los aromas utilizados por las marcas son deseados por los clientes para ser utilizados en sus espacios personales, encontramos marcas que venden como un producto más su aroma (Alzate y Lujan, 2017).

Finalmente, el tercer objetivo se analizó empíricamente mediante los datos presentados en las Figuras 7, 8 y 9, tal que se pudo constatar que el sentido del olfato ocupa el segundo lugar, por niveles de importancia, al momento de comprar, lo que llama la atención para que se tomen decisiones estratégicas de ventas tomando en consideración el marketing olfativo. Esta conclusión toma fuerza cuando en el análisis de datos se pudo ver que el $89 \%$ de los encuestados respondieron afirmativamente para que los productos y marcas tuvieran un aroma de identificación. En Colombia ya se encuentran algunos sectores utilizando las estrategias de marketing olfativo, sin 
embargo, el proceso de medición del merchandising olfativo es deficiente, en algunos casos la estrategia pierde fuerza y se queda solo como aromatizante de espacios (Grisales, 2019). Se puede evidenciar una aceptación muy alta por parte de los encuestados, de manera que sería una oportunidad para la empresas creadoras y distribuidoras de marketing olfativo, mejorar sus estrategias de comunicación para que más sectores se vinculen y realicen merchandising olfativo para sus marcas. Los sectores de hotelería, entretenimiento, retail y salud son algunos de los que han logrado vincular dicha estrategia y que ha permanecido en el tiempo (Alzate y Lujan, 2017).

\section{Referencias}

(1) Álvarez, R. (2011). Neuromarketing: seducir al cerebro con inteligencia para ganar en tiempos exigentes. Editorial Pearson.

(2) Alzate, M. y Lujan, D. A. (2017). PSICOLOGÍA Y MARKETING OLFATIVO. Una exploración desde la psicología sobre el impacto que ejerce en el consumidor el uso de odotipos. [Trabajo de Grado, Universidad de Antioquia] http://bibliotecadigital.udea.edu.co/bitstream/10495/14338/1/AlzateSandra_2017_Psicologia MarketingOlfativo.pdf

(3) Bonadeo, M. J. (2005). ODOTIPO Historia natural del olfato y su función en la identidad de marca. Editorial Universidad Austral.

(4) Bushdid, C., Magnasco, M. O., Vosshall, L. B. \& Keller, A. (2014). Humans can discriminate more than 1 trillion olfactory stimuli. Science, 343(6177), 1370-1372. https://doi.org/10.1126/science.1249168

(5) De Garcillán, M. (2015). Persuasión a través del marketing sensorial y experiencial. Opción, 31(2), 463-478. https://www.redalyc.org/pdf/310/31045568027.pdf

(6) Díez, C. M. (2013). Marketing Olfativo. ¿Qué olor tienes en mente? [Trabajo de Grado, Universidad de León]. https://buleria.unileon.es/handle/10612/2828

(7) Gómez, C. y García, M. (2012). Marketing sensorial: Cómo desarrollar la atmósfera del establecimiento comercial. Revista Distribución y Consumo. (122), 30-39. https://www.mercasa.es/media/publicaciones/196/1336046531_pag_030-040_Gomez.pdf

(8) Gómez, I. (2017). Marketing olfativo: de la emoción a la decisión. [Trabajo de Grado, Universidad de Valladolid]. http://uvadoc.uva.es/bitstream/handle/10324/24106/TFG- 
N.649.pdf?sequence $=1 \&$ is Allowed $=\mathrm{y}$

(9) Grisales, C. P. (2019). El marketing olfativo como posicionamiento de marcas. Tendencias, 20(2), 69-92. https://doi.org/10.22267/rtend.192002.123

(10) Hernández, M., Tomaseti, E. y Miranda, E. (2016). Marketing olfativo: la influencia del olor sobre la memoria. Anuario de Jóvenes Investigadores, 9(9), 244-247. http://hdl.handle.net/10317/5934

(11) Krishna, A. (2012). An integrative review of sensory marketing: Engaging the senses to affect perception, judgment and behavior. Journal of Consumer Psychology, 22(3), 332-351. https://doi.org/10.1016/j.jcps.2011.08.003

(12) Lersch, P. (1966). La estructura de la personalidad. Editorial Scientia.

(13)Lindstrom, M. (2005). Broad sensory branding. Journal of Product \& Brand Management, 14(2), 84-87. https://doi.org/10.1108/10610420510592554

(14)López, C. (2017). Marketing sensorial: análisis de la percepción del consumidor en Starbucks. [Trabajo de Grado, Universidad de Sevilla]. https://idus.us.es/xmlui/handle/11441/66369

(15) Martineau, P. (1958). The personality of the retail store. Harvard Business Review, 36(1), 4755.

(16) Palacios, P. (2016). ¿A qué huelen las marcas? marketing olfativo: potencial y casos de marcas que crean y usan olores para crear experiencias. [Trabajo de Grado, Universidad de Sevilla]. https://idus.us.es/handle/11441/52710

(17) Rodríguez, M. A. (2018). Odotipo: El aroma que identifica a la marca. [Trabajo de Grado, Universidad Peruana de Ciencias Aplicadas]. http://hdl.handle.net/10757/625508

Cómo citar este artículo: Grisales, C., Hernández, E. y Montenegro, E. (2021). Percepción del consumidor sobre el impacto del merchandising olfativo como estrategia del marketing sensorial. Tendencias, 22(2), 1-25. https://doi.org/10.22267/rtend.212202.166 